\title{
Cytochrome c Release and Caspase Activation in Traumatic Axonal Injury
}

\author{
András Büki,, ${ }^{1,2}$ David O. Okonkwo, ${ }^{1}$ Kevin K. W. Wang, ${ }^{3}$ and John T. Povlishock ${ }^{1}$ \\ ${ }^{1}$ Department of Anatomy, Medical College of Virginia, Virginia Commonwealth University, Richmond, Virginia 23298-0709, \\ 2Department of Neurosurgery, Medical School of Pécs, Pécs, H-7623, Hungary, and ${ }^{3}$ Department of Neuroscience \\ Therapeutics, Parke-Davis Pharmaceutical Research, Division of Warner-Lambert Company, Ann Arbor, Michigan 48105
}

Axonal injury is a feature of traumatic brain injury (TBI) contributing to both morbidity and mortality. The traumatic axon injury (TAl) results from focal perturbations of the axolemma, allowing for calcium influx triggering local intraaxonal cytoskeletal and mitochondrial damage. This mitochondrial damage has been posited to cause local bioenergetic failure, leading to axonal failure and disconnection; however, this mitochondrial damage may also lead to the release of cytochrome c (cyto-c), which then activates caspases with significant adverse intraaxonal consequences. In the current communication, we examine this possibility.

Rats were subjected to $\mathrm{TBI}$, perfused with aldehydes at 15-360 min after injury, and processed for light microscopic (LM) and electron microscopic (EM) single-labeling immunohistochemistry to detect extramitochondrially localized cytochrome c (cyto-c) and the signature protein of caspase-3 activation (120 kDa breakdown product of $\alpha$-spectrin) in TAI. Combinations of double-labeling fluorescent immunohisto- chemistry (D-FIHC) were also used to demonstrate colocalization of calpain activation with cyto-c release and caspase-3-induction.

In foci of TAl qualitative-quantitative LM demonstrated a parallel, significant increase in cyto-c release and caspase-3 activation over time after injury. EM analysis demonstrated that cyto-c and caspase-3 immunoreactivity were associated with mitochondrial swelling-disruption in sites of TAI. Furthermore, D-IFHC revealed a colocalization of calpain activation, cyto-C release, and caspase- 3 induction in these foci, which also revealed progressive $\mathrm{TAl}$.

The results demonstrate that cyto-c and caspase- 3 participate in the terminal processes of TAl. This suggests that those factors that play a role in the apoptosis in the neuronal soma are also major contributors to the demise of the axonal appendage.

Key words: traumatic axonal injury; spectrin; calpain; caspase; cyto-c; axolemma; calcium; mitochondrial membrane permeability transition
Traumatic axonal injury (TAI) accounts for at least $35 \%$ of the morbidity and mortality of traumatic brain injury (TBI) patients without space-occupying lesions and also contributes to the mortality and morbidity caused by focal brain injuries (Gennarelli et al., 1982). Studies over the last two decades have demonstrated that the classical manifestation of TAI, axonal disconnection and the formation of an "axonal retraction ball" as described previously (Strich, 1956, 1961; Adams et al., 1977, 1980; Adams, 1982), is the result of several pathological processes that gradually evolve from subtle focal axonal perturbations to ultimate axonal disconnection (Povlishock et al., 1983). Initiating these evolving events are focal changes in axolemmal permeability involving mechanoporation of the axolemma evoked by the tear-shearing forces of the injury (Pettus et al., 1994; Povlishock and Pettus, 1996; Maxwell et al., 1997; Povlishock et al., 1997). It has been posited that this leads to the influx of calcium, and the correctness of this assumption has been confirmed in our laboratory by the demonstration of the activation of calpain-mediated spectrin pro-

Received Aug. 26, 1999; revised Jan. 28, 2000; accepted Jan. 31, 2000.

This work was supported by National Institute of Health Grant NS 20193 and the Martin Rodbell Fellowship from Philip Morris USA. We thank Robert Siman for the provision of the Ab38 antibody. We also thank Susan Walker, Lynn Davis, and Thomas Coburn for their excellent technical support and Robert Hamm for his statistical advice.

Correspondence should be addressed to John T. Povlishock, Professor and Chair, Department of Anatomy, Medical College of Virginia, Virginia Commonwealth University, Richmond, VA 23298-0709. E-mail: jpovlish@hsc.vcu.edu.

Copyright (C) 2000 Society for Neuroscience $0270-6474 / 00 / 202825-10 \$ 15.00 / 0$ teolysis (CMSP), first confined to the subaxolemmal and perimitochondrial spectrin domains and then, over time, advancing deeper within the axon cylinder (Büki et al., 1999a). Concomitant with this calpain activation, the related axonal cytoskeleton and organelles also showed change consistent with calcium overloading as reflected in neurofilament sidearm modification and compaction, microtubular loss, and local mitochondrial swelling with disruption of their cristae, with the suggestion that such mitochondrial perturbation was a terminal event in the local demise of the axon. In this context, it was recognized that the observed mitochondrial swelling was entirely consistent with a calciuminduced opening of the mitochondrial membrane permeability transition (MPT) pore. Recently, our laboratory has provided some additional support for this assumption through the use of cyclosporin-A, a known inhibitor of the MPT pore, which provided not only profound mitochondrial protection but also a significant blunting of the above described CMSP and related cytoskeletal changes (Büki et al., 1999b; Okonkwo and Povlishock, 1999; Okonkwo et al., 1999). Based on these observations, we initially hypothesized that the preservation of mitochondria and their bioenergetic capacity were integral to axonal survival. However, despite the credibility of our argument, this premise did exclude the potential that this mitochondrial preservation also blunted the release of damaging factors, such as cyto-c, which when interfaced with other intraaxonal factors, could initiate an agonal cascade of caspase activation (Cai et al., 1998; Susin et al., 1998, 1999a,b; Gorman et al., 1999; Krajewski 
et al., 1999; Martinou et al., 1999; Uehara et al., 1999) with catastrophic consequences for the axon. Although our understanding of cyto-c and caspase cascade is well established in the neuronal somata wherein these agents trigger apoptosis that contributes to both ischemic (Krajewski et al., 1999) and traumatically induced neuronal injury (Rink et al., 1995; Clark et al., 1997; Yakovlev et al., 1997; Conti et al., 1998; Fox et al., 1998; McIntosh et al., 1998; Pike et al., 1998a,b; Morita-Fujimura et al., 1999), their involvement in the axon cylinder is, at present, unknown.

In the present communication, we explore this very issue through the use of immunohistochemical approaches to demonstrate for the first time at the electron microscopic (EM) level the potential for mitochondrial cyto-c release in concert with spatially and temporally related caspase activation. Also, in select cases, we evaluate the relationship existing between our previously described activation of CMSP and any change in cyto-c release and related caspase activation. Via these approaches, we provide evidence for the existence of a hierarchy of events wherein CMSP precedes cyto-c release and caspase activation. We also demonstrate that, comparable with the neuronal somatic caspase-apoptotic cascade, caspase activation within the axonal cylinder most likely constitutes the terminal event in its demise.

\section{MATERIALS AND METHODS}

Induction of traumatic brain injury. In the present study, we used a rodent model of impact acceleration head injury that has been described in detail previously (Foda and Marmarou, 1994; Marmarou et al., 1994). In all, 30 Sprague Dawley rats (Charles River Laboratories, Raleigh, NC) weighing 365-398 gm were used for the experiments; 25 were subjected to traumatic brain injury, and 5 underwent sham operation. For the induction of anesthesia, each animal was exposed to $4 \%$ isoflurane (Iso Flo; Abbott Laboratories, North Chicago, IL) in a bell jar for $5 \mathrm{~min}$ and then intubated and ventilated with a mixture of $1-2 \%$ isoflurane in $30 \%$ $\mathrm{O}_{2}$ and $70 \% \mathrm{~N}_{2} \mathrm{O}$. Next, the skull between the coronal and lambdoid sutures was exposed following a midline scalp incision. A metallic diskshaped helmet of $10 \mathrm{~mm}$ diameter was firmly attached to this point of the skull using dental acrylic. The animal was placed in a prone position on a foam bed with the metallic helmet centered under the edge of a Plexiglas tube. The rat was prevented from falling by two belts secured to the foam pad. Brass weights weighing $450 \mathrm{gm}$ were allowed to fall from a height of $200 \mathrm{~cm}$ through the Plexiglas tube directly to the metallic disk fixed to the animal's skull, a setting that does not produce either cerebral contusion or subdural hemorrhage. After injury, the animal was immediately ventilated with $100 \% \mathrm{O}_{2}$. The helmet was removed, and the skull was examined for any sign of fracture which, if found, disqualified the animal from further evaluation. The scalp wound was sutured, with the animal remaining on artificial ventilation until it regained spontaneous breathing, and then the animal was killed at the predetermined survival periods of 15, 30, $60 \mathrm{~min}, 3 \mathrm{hr}$, and $6 \mathrm{hr}$ after injury. Five sham-injured animals (one per each survival period) were treated in the same manner but were not injured.

Physiological assessments. The respiratory status was monitored through pulse oximetry via the footpad and/or the tongue. Additionally, brain temperature was monitored by a temporalis muscle probe, and core temperature was determined by a rectal probe.

Immunohistochemistry. At the designated survival time, the rats were reanesthetized with an overdose of sodium pentobarbital and transcardially perfused with $4 \%$ paraformaldehyde and $0.1 \%$ glutaraldehyde in Millonig's buffer. Brains were removed and immersed in the same fixative overnight (16-18 hr). On the basis of our previous observations concerning the topography of diffusely injured axons in rat brain (Povlishock et al., 1997), a midline, 5-mm-wide block of the brain was removed using a sagittal brain blocking device to include the region extending from the interpeduncular fossa to the first cervical segment. The tissue was sectioned with Vibratome Series 1000 (Polysciences Inc., Warrington, PA) at a thickness of $30 \mu \mathrm{m}$ and collected in $0.1 \mathrm{M}$ phosphate buffer. The sections were collected in five groups in a semi-serial manner, rinsed three times for $10 \mathrm{~min}$ in PBS, placed in a sodium-citrate buffer, $\mathrm{pH}$ 6.0, and transferred to a programmable, magnetron-powered $900 \mathrm{~W}$, PELCO
3460 microwave oven (Ted Pella, Redding, CA). This laboratory microwave oven equipped with a load cooler and a computerized temperature monitoring system was operated with $70 \%$ energy cycling over a $2 \times 5$ min period, during which tissue temperature was never allowed to rise above $40^{\circ} \mathrm{C}$. This approach was based on our recent finding (Stone et al., 1999) that the use of microwave energy without the generation of significant heat significantly enhances immunoreactivity (IR) but suppresses background immunoreactivity. This controlled temperature approach also allowed for the preservation of excellent ultrastructural immunohistochemical detail, unlike traditional approaches that may cause tissue damage via the generation of significant heat (Stone et al., 1999). After microwave processing, sections were treated in one of the following manners. The first two groups of tissue were processed for qualitative and quantitative LM (vide infra) and EM analysis, respectively, using single labeling with an antibody targeting cyto-c only upon its release from the mitochondrial intermembrane space (Fujimura et al., 1998, 1999). The third and fourth groups of tissue were reacted with the spectrin breakdown product (SBDP)-120 antibody for qualitative and quantitative LM (vide infra) and EM analysis, respectively. This antibody is known to target the $120 \mathrm{kDa}$ breakdown product of brain $\alpha$-spectrin (fodrin) (Nath et al., 1998; Pike et al., 1998a,b; Wang et al., 1998a; Z hao et al., 1999). The stable $120 \mathrm{kDa}$ SBDP is a "signature protein," exclusively produced on proteolytic processing of brain $\alpha$-spectrin by caspase-3 and, as such, SBDP-120-IR constitutes a reliable indicator of the activation of the caspase death cascade (Wang, 1999). The fifth group of sections was processed for double-labeling fluorescent immunohistochemistry (FIHC). Here, we used two additional antibodies in concert with the use of the above described cyto-c and SBDP-120. Specifically, we used the Ab38 antibody (AB) targeted to the N-terminal fragment of the $150 \mathrm{kDa}$ breakdown product of the $\alpha$ subunit of brain spectrin produced solely upon its cleavage by the calcium-activated protease calpain (Siman et al., 1989; Roberts-Lewis et al., 1994). As we have described previously, CMSP is pivotally involved in the pathogenesis of TAI. Thus, in the current investigation, the Ab38 antibody served as an early, sensitive marker of TAI (Büki et al., 1999a) and also provided important comparative data as to how one group of cysteine proteases (calpains) relates to another (caspases) in the ongoing pathology of TAI (Büki et al., 1999b,c; Okonkwo et al., 1999). In this fluorescent double-labeling protocol, we also used the p20 antibody, which recognizes the activated form of the caspase- 3 enzyme that targeted another aspect of caspase activation. The use of the p20 antibody provided additional confirmation of any potential caspase activation and also provided an internal control for generalized caspase activation. In these fluorescence double-labeling studies, we used one of the following primary antibody combinations: colocalization of AB with cyto-c-AB, cyto-c-AB with SBDP-120 AB, SBDP-120 with Ab38, and SBDP-120 AB with p20 AB.

Light microscopic and EM single-labeling protocol. After rinsing in PBS, sections from the first four groups were preincubated for $35 \mathrm{~min}$ with $0.2 \%$ Triton X-100 (Sigma, St. Louis, MO) in 10\% normal horse serum (NHS) (for cyto-c) or normal goat serum (NGS) (for SBDP-120) (Sigma) in PBS. After two quick rinses in PBS containing 1\% NHS or NGS, the sections were incubated overnight in mouse cyto-c antibody (Boehringer Mannheim, Mannheim, Germany) at a dilution of 1:1000 or in rabbit SBDP-120 antibody at a dilution of 1:3000. After three washes for $10 \mathrm{~min}$ each in PBS containing 1\% NHS or NGS, sections were incubated in biotinylated anti-mouse or anti-rabbit Ig derived from horse or goat, respectively (diluted 1:200 in 1\% NHS-PBS or NGS-PBS) (Vector Laboratories, Burlingame, CA) for $60 \mathrm{~min}$, followed by three rinses for $10 \mathrm{~min}$ each in PBS. After incubation in an avidin biotin-peroxidase complex (ABC standard Elite kit, at a dilution of 1:100; Vector Laboratories) and rinsing in PBS and $0.1 \mathrm{M}$ phosphate buffer (three times for 10 min and two times for $10 \mathrm{~min}$, respectively), sections were processed for visualization of the immunohistochemical complex using $0.05 \%$ diaminobenzidine (DAB) (Sigma) and $0.01 \%$ hydrogen peroxide in $0.1 \mathrm{M}$ phosphate buffer. Sections from groups one and three were mounted, cleared, and coverslipped (Cytoseal 60; Thomas Scientific, Swedesboro, $\mathrm{NJ}$ ) for routine LM examination. LM photomicrographs were captured using a Nikon (Tokyo, Japan) Eclipse 800 photomicroscope equipped with a Sony (Tokyo, Japan) Catseye digital camera. Sections from groups two and four were osmicated, dehydrated, and flat-embedded between plastic slides in medcast resin (Ted Pella, Redding, CA) following the same sequence of procedures described above. Immunopositive foci were trimmed, mounted on plastic studs, and sectioned using an LKB-Wallac (Gaithersburg, MD) Ultratome at a thickness of 70-100 $\mathrm{nm}$ thin sections. Thin sections were picked up onto Formvar-coated, slot grids and then 
stained in 5\% uranyl acetate in 50\% methanol for $2 \mathrm{~min}$ and $0.5 \%$ lead citrate for $1 \mathrm{~min}$. Ultrastructural analysis was performed using a Jeol1200 electron microscope (Jeol, Peabody, MA).

Immunofluorescence double-labeling protocol. To increase the sensitivity of the FIHC and reduce antibody use, we used the tyramide signal amplification (TSA) method (Hunyady et al., 1996; van Gijlswijk et al., 1997; Büki et al., 2000) in conjunction with routine FIHC techniques. In this approach, the fifth group of sections underwent the same basic IHC steps described above, including microwave antigen retrieval, PBS rinsing, and incubation for $35 \mathrm{~min}$ with PBS containing 10\% NGS (Vector Laboratories) and 0.2 Triton X-100. The sections were then incubated overnight in one of the following combinations: (1) Ab38 (rabbit, polyclonal, 1:6000) with cyto-c (mouse, monoclonal, 1:300); (2) cyto-c (mouse, monoclonal, 1:300) with SBDP-120 (chicken, polyclonal, 1:5000); (3) SBDP-120 (chicken, polyclonal, 1:5000) with Ab38 (rabbit, polyclonal, 1:1000); and (4) SBDP-120 (chicken, polyclonal, 1:5000) with p20 (rabbit, polyclonal, 1:500). The secondary antibodies-TSA labels were respectively applied in a darkroom as follows: (1) biotinylated anti-rabbit goat Ig (Vector Laboratories) with Alexa 594 fluorescence anti-mouse goat Ig (diluted 1:200 in 1\% NGS; Molecular Probes, Eugene, OR), followed by incubation in the Vector Laboratories ABC kit (1:100), and then reacted with the Fluorochrome-TSA-kit (FITC-TSA, 1:200); (2) Alexa 594 fluorescence anti-mouse goat Ig (Molecular Probes) with biotinylated anti-chicken goat Ig (diluted 1:200 in 1\% NGS; Vector Laboratories), followed by incubation in the Vector ABC kit (1:100), and then reacted with the Fluorochrome-TSA-kit (FITC-TSA, 1:200); (3) biotinylated anti-chicken goat Ig (Vector Laboratories) with Alexa 594 fluorescence anti-rabbit goat Ig (diluted 1:200 in 1\% NGS; Molecular Probes), followed by the Vector Laboratories ABC kit (1:100), and then reacted with the Fluorochrome-TSA-kit (FITC-TSA, 1:200); and (4) biotinylated anti-chicken goat Ig (Vector Laboratories) with Alexa 594 fluorescence anti-rabbit goat Ig (diluted 1:200 in 1\% NGS; Molecular Probes), followed by the Vector Laboratories ABC kit (1:100), and then reacted with the Fluorochrome-TSA-kit (FITC-TSA, 1:200). Finally, all sections were mounted on gelatin-coated slides and coverslipped using Gel/Mount (Blomeda Corp., Foster City, CA), and the coverslips were sealed with nail polish. Digital images were captured using a Nikon Eclipse 800 fluorescence photomicroscope equipped with a Sony Catseye digital camera. For data analysis and identification of double-labeled immunofluorescence axonal profiles, digital comparisons of identical fields were accomplished through the use of Adobe Systems (San Jose, CA) Photoshop 5.0 software.

Immunohistochemical controls. All the IHC procedures applied in this study complied with the basic criteria of specificity and sensitivity (Petrusz et al., 1976, 1980). To this end, the optimal specific staining/ background ratio was characterized using several dilution protocols. All IHC reactions were completed with incubation of tissue sections in the absence of either the primary or secondary antibodies; in the case of FIHC staining procedures, this regimen was supplemented with sequential incubation in the primary and secondary antibodies and with incubation without TSA or in the TSA kit alone. To achieve further rigor, selected sections were incubated with working dilutions of the cyto-c antibody previously preadsorbed with $10 \mu \mathrm{g} / \mathrm{ml}$ concentration of cyto-c (Sigma) or SBDP-120 antibody previously preadsorbed with $1 \mu \mathrm{g} / \mathrm{ml}$ concentration of SDEALC-peptide. To date, the Ab38 antibody has been well characterized via immunoblot and preadsorbtion protocols (Siman et al., 1989; Roberts-Lewis et al., 1994; Saatman et al., 1996a; Posmantur et al., 1997). Similarly, the p20 antibody has been thoroughly characterized via immunoprecipitation and immunoblotting by R \& D Systems (Minneapolis, MN).

Digital data acquisition and image analysis. All sections from groups 1 and 3 were examined with a Nikon Eclipse 800 light microscope to quantitatively analyze and determine the temporal characteristics of cyto-c release and caspase- 3 activation in traumatically injured axonal profiles. In both groups, all sections containing the medullary pyramid were selected ( $n=4-7$ per group per animal). Using a $4 \times$ objective lens and a $2.5 \times$ intermediate lens with the aid of an ocular micrometer, the distance between the most cranial ("A") and caudal ("B") point of the medullary pyramid was determined ("d"). The microscope was centered over the corticospinal tract (CSpT) at the junction of the middle and distal third of "d". Next, a $20 \times$ objective was centered at this point, and a digitized image was captured. The $20 \times$ objective was then centered over the medial longitudinal fasciculus (MLF) at the level of point "B", and a second image was captured. Both the CSpT and the MLF were targeted for analyses because our previous studies had shown that these foci consistently contained numerous traumatically damaged axons (Povlishock et al., 1997). The images obtained in this manner were analyzed with a computer-assisted image analysis system (Imaging Research, St. Catherine's, Ontario, Canada) in a blinded manner. In both the CSpT and the MLF, a $100,000 \mu \mathrm{m}^{2}$ grid $(200 \times 500)$ was superimposed on the captured image, and those immunoreactive axonal profiles over $5 \mu \mathrm{m}$ in diameter were counted. The total number of immunopositive axonal profiles within these regions were expressed as the density of immunopositive axons per square millimeter.

Statistical analysis. Mean density values of immunoreactive axonal segments were determined using two randomly selected sections from each rat and each anatomical region tested. Mean density values for each anatomical region (CSpT and MLF) were analyzed separately in a completely randomized design in which time constituted the independent variable. In addition to the use of an overall ANOVA, additional simple effect and cell comparison were accomplished by Scheffe's post hoc comparisons. Differences were considered significant at $p<0.05$.

\section{RESULTS}

\section{Sham control}

With either routine LM IHC or FIHC, rats undergoing sham injury did not display any immunoreactivity that could complicate data analysis. This finding was consistent with the fact that, in the sham controls, the axons would not be expected to manifest any form of reactive change.

\section{Physiological data}

Consistent with our previous experience with the impact acceleration brain injury model, none of the physiological parameters monitored demonstrated significant change from the well established physiological baselines.

\section{Routine light microscopic immunohistochemistry: qualitative analysis}

Qualitative analysis of LM IHC revealed that focally injured axonal segments displayed cyto-c-IR and the caspase-linked SBDP-120-IR in the pontomedullary segment of the CSpT and the MLF within 15 min of injury (Fig. $1 A, C$, respectively). At this time point, the overall number of both the cyto-c-IR and SBDP120-IR axonal profiles appeared modest. However, with increasing survival (30-360 $\mathrm{min})$, the number of the immunoreactive axonal profiles appeared dramatically increased on qualitative assessment (Fig. 1B,D). For all survival times and anatomical regions, the morphological characteristics of cyto-c-IR and SBDP-120-IR axonal profiles were remarkably similar. Consistent with our previous descriptions using other IHC markers of TAI, the scattered IR axonal segments at 15 min after injury displayed focal swelling and vacuolization (Fig. $1 \mathrm{~A}, \mathrm{C}$ ), whereas with more prolonged postinjury survival, these axonal segments displayed progressive swelling, pronounced vacuolization, disconnection, and fragmentation (30-360 min) (Fig. 1B,D). Although parallel analyses of somatic immunoreactivity were beyond the scope of the present study, it is of note that with the above described IHC protocols, we did not find consistent neuronal immunostaining in the brainstem nuclei anatomically related to those foci showing TAI.

\section{Routine light microscopic immunohistochemistry: quantitative analysis}

The mean density of cyto-c-IR and caspase-associated SBDP120-IR axonal profiles for the CSpT and the MLF over the five postinjury survival times are presented in Figures 2 and 3, respectively. The results of ANOVA indicate a main effect of postinjury survival time on the density of cyto-c-IR axonal profiles in the CSpT $\left(F_{(4,20)}=67.715, p<0.001\right)$, as well as in the $\operatorname{MLF}\left(F_{(4,20)}=31.969, p<0.001\right)$. Scheffe's post hoc comparisons 


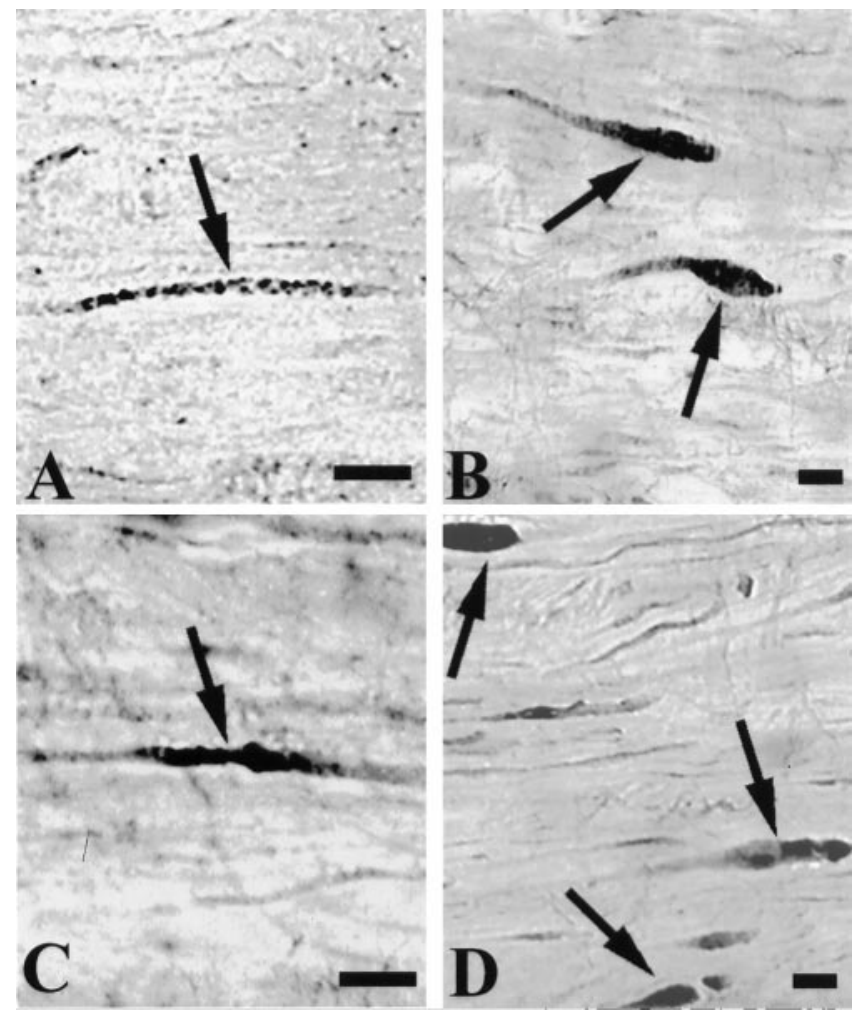

Figure 1. Axons displaying cyto-c-IR $(A, B)$ and SBDP-120-IR $(C, D)$ in rat medial longitudinal fasciculus (arrows). At 15 min after injury $(A, C)$, the axonal segments appear swollen $(A, C)$, and at $360 \mathrm{~min}$ after injury, both cyto-c- and SBDP-120-IR axonal profiles display evidence of imminent disconnection $(B, D)$. Scale bar, $15 \mu \mathrm{m}$.

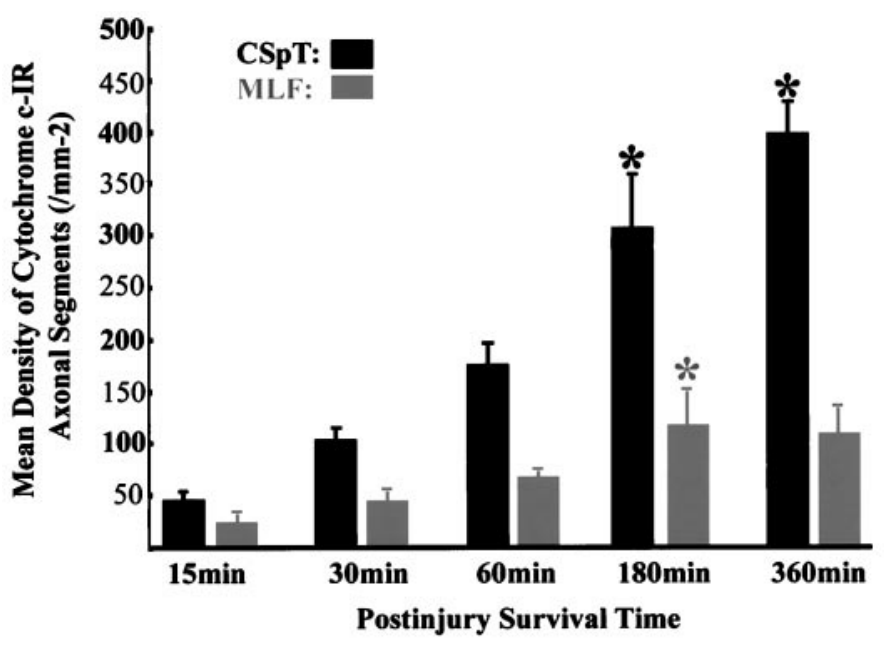

Figure 2. Bar charts representing the mean densities of immunopositive axons displaying cyto-c immunoreactivity in the CSpT (black) and the MLF ( gray) at various postinjury survival times after impact acceleration injury. Error bars indicate SEM, and the asterisks indicate statistical significance $(p<0.05)$ between consecutive survival times. (Significant changes between non-neighboring survival times are not indicated.)

indicated a significant increase in the mean density of cyto-c-IR axonal profiles between consecutive survival times analyzed in the CSpT between $60 \mathrm{~min}$ and $3 \mathrm{hr}(p<0.01)$ and between 3 and $6 \mathrm{hr}(p<0.04)$. In the MLF a significant increase in cyto-c-IR was seen between $60 \mathrm{~min}$ and $3 \mathrm{hr}(p<0.01)$ (Fig. 2).

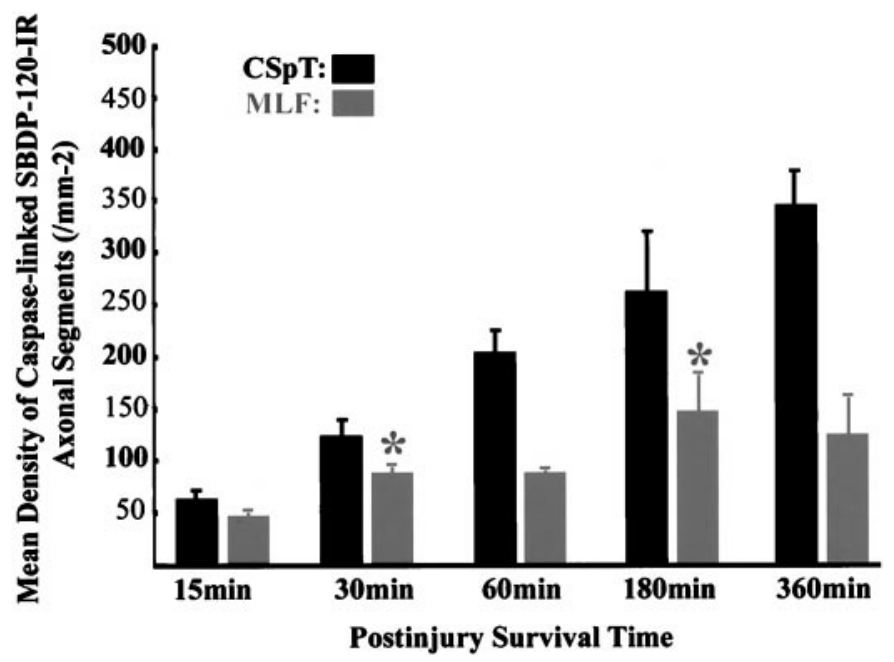

Figure 3. Bar chart of the mean densities of immunopositive axons displaying caspase-linked SBDP-120- immunoreactivity in the CSpT (black) and the MLF ( gray) at various postinjury survival times after impact acceleration injury. Error bars indicate SEM, and the asterisks indicate statistical significance $(p<0.05)$ between consecutive survival times. (Significant changes between non-neighboring survival times are not indicated.)

Furthermore, ANOVA also proved a main effect of postinjury survival time on the density of caspase-linked SBDP-120-IR axonal profiles in the $\operatorname{CSpT}\left(F_{(4,20)}=41.986, p<0.001\right)$ and also in the $\operatorname{MLF}\left(F_{(4,20)}=19.156, p<0.001\right)$ (Fig. 3). Scheffe's post hoc comparisons indicated a significant increase in the mean density of cyto-c-IR axonal profiles between consecutive survival times analyzed in the MLF between 15 and $30 \min (p<0.05)$ and between 60 and $180 \min (p<0.01)$.

\section{Ultrastructural analysis}

Immunoelectron microscopy demonstrated that both cyto-c-IR and SBDP-120-IR axonal segments displayed the previously described ultrastructural characteristics of traumatic axonal injury, including focal cytoskeletal alterations (neurofilament compaction and microtubular loss) axolemmal infoldings, and altered structure of the myelin sheet. In those scattered axonal profiles displaying cyto-c immunoreactivity within 15-30 min after injury, the electron-dense DAB chromogen was confined to either the surface of the mitochondria or in close proximity frequently obscuring mitochondrial fine structural details (Fig. 4A, C). However, in fortuitous sections, the immunoreactivity was associated with mitochondria, showing some form of change in terms of mitochondrial swelling and/or disruption of their cristae. Importantly, these abnormal mitochondria were localized exclusively to those axonal segments showing other forms of well characterized reactive change, such as neurofilament compaction, microtubular loss, and other ultrastructural abnormalities listed above. For example, it was quite common to see isolated damaged axonal profiles with immunoreactive mitochondria in fields of other unaltered axons showing no mitochondrial immunoreactivity (Fig. 4A,B). This rather remarkable finding argues for the sensitivity-specificity of the antibody, which is confined only to damaged axonal segments with altered mitochondria that are capable of releasing cyto-c from their inner matrix. With prolonged postinjury survival (60-360 $\mathrm{min}$ ), cyto-c-IR was once again found in relation to damaged mitochondria consistently localized to damaged-reactive axonal segments (Fig. 4D). Typically, with 

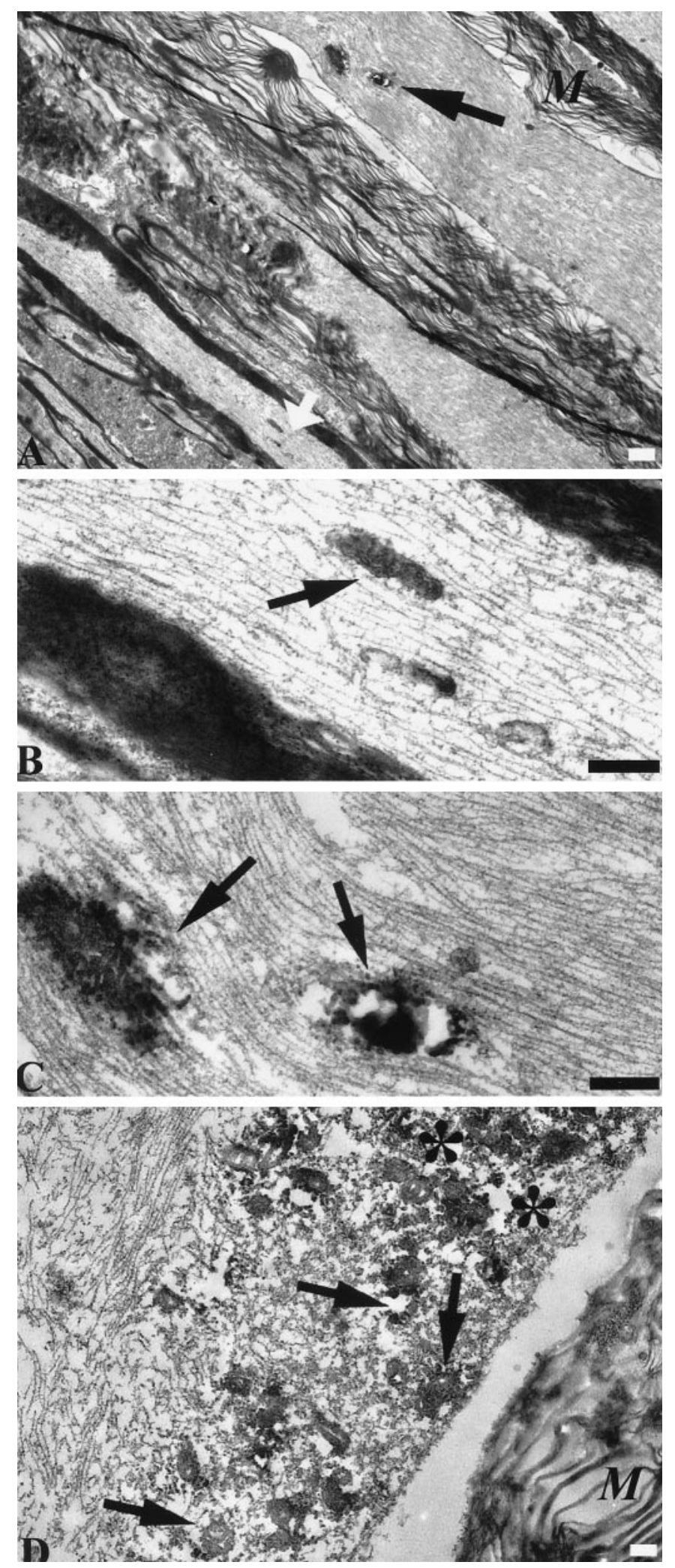

Figure 4. Electron micrographs of axonal profiles demonstrating cytoc-IR $(A-D)$. Overview of a segment of the corticospinal tract 30 min after injury $(A)$. The short white arrow marks an uninjured axonal segment illustrated in $B$ in high-power magnification, and the long black arrow points to a portion of the traumatically altered axonal profile enlarged in $C$. Note the loosening of the myelin sheet around the injured axonal segment $(M)$. Uninjured axonal profiles $(B)$ display normal interfilament distance, unaltered mitochondrial structure (arrow), and the lack of increased survival time, the immunoreactive mitochondria were associated with more overt forms of cytoskeletal changes reflected in disarray of the compacted and partially digested neurofilaments with pooling of some immunoreactive mitochondria, consistent with a disruption of axoplasmic transport long associated with the progression of TAI (Povlishock, 1992). In parallel with this cyto-c IR, axonal segments harvested from adjacent sections manifesting the above described TAI pathology also revealed clear evidence of caspase activation reflected in the presence of SBDP-120 immunoreactivity (Fig. 5A-C).

Thus, although cyto-c-IR and SBDP-120-IR were visualized in adjacent sections, their ultrastructural localization only to sites of TAI suggested that both antibodies target the same reactive axonal pathological process. The caspase-linked immunoreactivity (SBDP-120) was confined to the cytoskeletal and perimitochondrial spectrin constituents, entirely consistent with the known distribution of brain spectrin within the axon (Fig. 5A) (Zagon et al., 1986; Goodman et al., 1995). Over time, this IR was associated with more dramatic cytoskeletal and mitochondrial change, including mitochondrial swelling and pooling, consistent with the above described pathology (Fig. 5B,C). Although a direct correlation existed between the presence of mitochondrial cyto-c-IR and mitochondrial-cytoskeletal SBDP-120-IR, the SBDP-120-IR allowed for an easier recognition of swollen-disrupted mitochondria than seen through the use of cyto-c-IR. This unanticipated finding can be explained that the cyto-c-IR reaction had a higher tendency to cover the mitochondria themselves.

\section{Double-labeling fluorescence immunohistochemistry}

Recognizing that colocalization of the antibodies and their immunohistochemical markers used in double-labeling FIHC could impair their relative penetration and thereby limit the validity of any quantitative analysis of the double-labeled axonal profiles, we used double-labeling FIHC exclusively as a qualitative arm of this study.

The anatomical localization and overall morphological characteristics of immunofluorescence axonal profiles were entirely consistent with that described in the previous passages, as well as in our previous communications; however, now they provided new data on their overall spatiotemporal characteristics (Fig. $6 A-L)$. In the early posttraumatic period (15-30 min after injury), CMSP-IR predominated with little suggestion of colocalization with cytochrome c, SBDP-120, or p20 immunoreactivity. Furthermore, CMSP-IR axons marked linear, less reactive immunoreactive axonal segments, particularly within the pyramidal tract, a finding consistent with our previous observation that CMSP delineates these axons in the initiating phases of TAI. Over time (60-360 min after injury), however, the predominate CMSP-IR was replaced by the consistent finding of CMSP-immunopositive profiles, which also colocalized cyto-c and SBDP-120 or p20 immunoreactivity (Fig. 6A-C,J-L). This colocalization of CMSP-IR and SBDP-120-IR provided evidence that traumati-

$\overline{\text { DAB deposition. Traumatically injured scattered axonal segments from }}$ the same field $(C)$ reveal neurofilament compaction and mitochondrial alteration with the electron-dense DAB chromogen partially covering the surface of the swollen mitochondria (arrows). Six hours after injury $(D)$, the cytoskeletal alterations, such as neurofilament compaction and digestion, as well as the modificaton of the myelin sheet $(M)$, is more obvious. Note the pooling of the mitochondria, many of which are disrupted or swollen (arrows). Also note the cyto-c immunoreactivity, which diff uses into the surrounding axoplasm (asterisks). Scale bar, $500 \mathrm{~nm}$. 

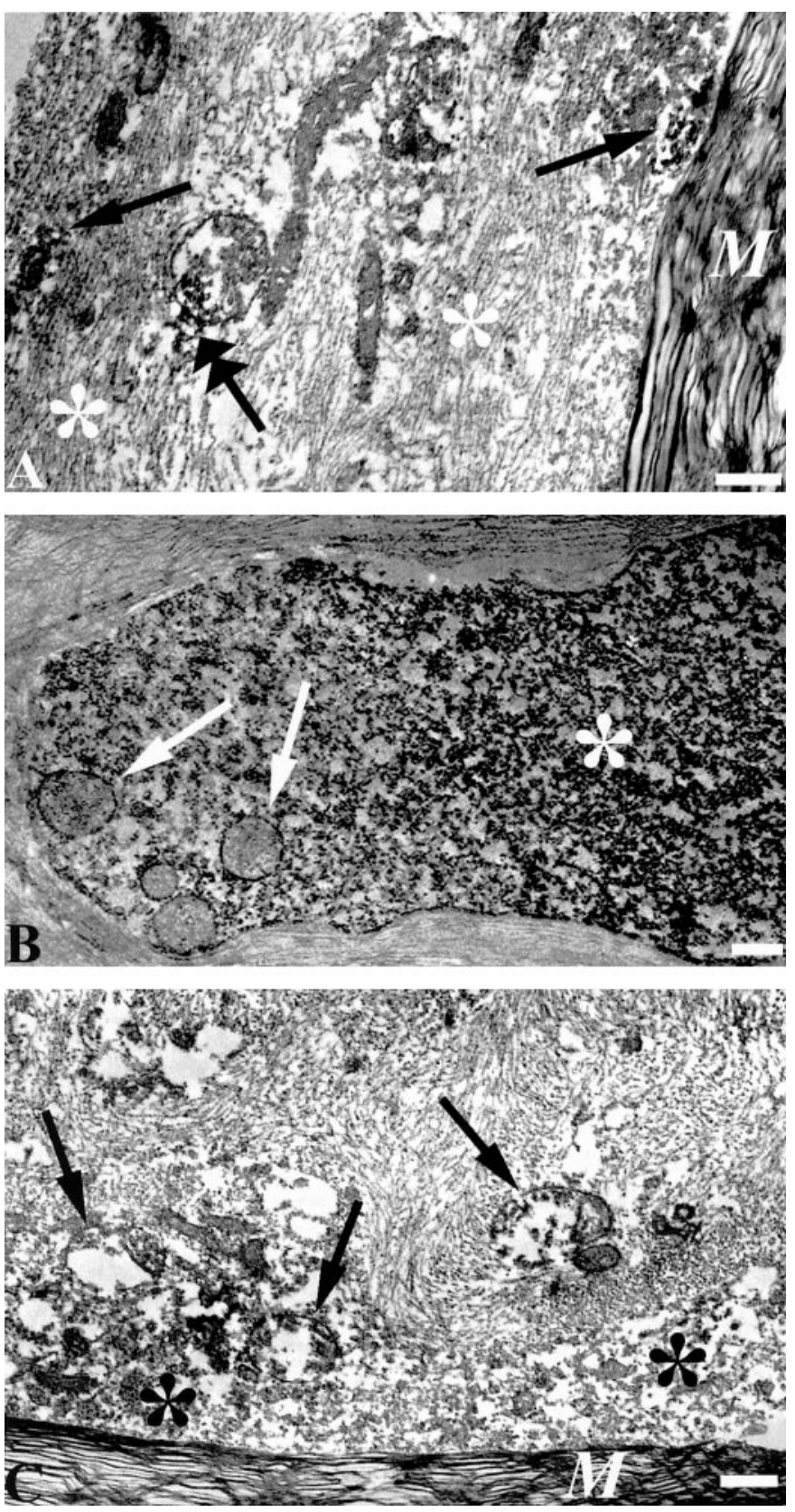

Figure 5. Electron micrograph of caspase-associated SBDP-120immunoreactive axonal profiles. At 30 min after injury $(A)$, the electrondense DAB reaction product is primarily associated with cytoskeletal elements (arrows) and the perimitochondrial domain (double arrow). Note a damaged swollen mitochondrion with partially ruptured cristae approximating normal-appearing mitochondria. Also, note the loosening of the myelin sheet $(M)$ and the reduction of interfilament distance (white asterisks). Three hours after injury ( $B$, unstained section), the cytoskeletal damage (and the related SBDP-120-IR) is more obvious (white asterisk), as is the mitochondrial swelling (arrows). At $6 \mathrm{hr}$ after injury $(C)$, note the cytoskeletal alterations and the severe mitochondrial damage (arrows) reflected in their pooling, swelling, and rupture. Also note the myelin alterations $(M)$ and the electron-dense DAB associated with damaged mitochondria and/or the disintegrated cytoskeletal elements (asterisk). Scale bar, $500 \mathrm{~nm}$.

cally injured axonal foci are prone to proteolytic modification and destruction by both members of the cysteine protease family, calpain and caspase (Fig. $6 J-L)$.

Furthermore, the fact that p20-IR (active caspase-3 fragment immunoreactivity) and SBDP-120-IR were colocalized in the same damaged axonal segments (Fig. 6G-I) gives additional credibility to our assumptions concerning the activation and the role of the caspase death cascade.

\section{Immunohistochemical controls}

The use of primary antisera preadsorbed according to the previously described protocol resulted in the lack of immunoreactivity, as well as the exclusion of either the primary or the secondary antibody from the IHC reaction. Gradual overdilution of the immunosera resulted in the dissipation and disappearance of the immunostaining. Simultaneous or sequential application of immunosera in the case of double-labeling FIHC resulted in equally powerful detection of both antigens. The TSA kit alone did not contribute to any specific immunohistochemical reaction that might have influenced the data analysis.

\section{DISCUSSION}

In the present study, we provide, for the first time, evidence that traumatically evoked mitochondrial alterations lead to cyto-c release and the activation of the caspase enzyme cascade in axons. Furthermore, we demonstrate that such cyto-c release and caspase activation are spatially and temporally connected to calcium-induced, calpain-mediated spectrin proteolysis, suggesting the interaction of the cysteine proteases in the pathogenesis of traumatic axonal injury. In addition to these novel observations, this study also confirms and extends our previous findings in traumatic brain injury, now providing a comprehensive picture of the pathobiological events ongoing in the traumatically injured axon from its inception to its terminal phases. Those intraaxonal events both assessed in our previous communications (Büki et al., 1999a; Okonkwo and Povlishock, 1999) and further evaluated in the current communication speak to the involvement of calcium acting through multiple, concurrent pathways, including calciumactivated neutral proteases, mitochondrial perturbation, cyto-c release and caspase activation. The observed mitochondrial perturbation is consistent with calcium overloading and opening of the MPT pore, which has been linked to calcium loading and implicated in the pathogenesis of several neuronal diseases (for review, see Cai et al., 1998; Lemasters et al., 1998; Montal, 1998; Siesjo et al., 1999), because excessive sequestration of calcium leads to the dissipation of the mitochondrial transmembrane potential and the opening of the MPT pore that permealizes the mitochondrial membrane for molecules $<1.5 \mathrm{kDa}$. The process leads to the uptake of water, mitochondrial swelling, and ultimate mitochondrial rupture (Zoratti and Szabo, 1995; Trost and Lemasters, 1996; Hirsch et al., 1998; Lemasters et al., 1998). Although we believe that our data, as well as others, speak to the likelihood of MPT, several recent papers have suggested that MPT in itself is not prerequisite for the release of cyto-c (Eskes et al., 1998; Jurgensmeier et al., 1998; Schendel et al., 1998). Whether or not this is the case in the current communication, however, is not of consequence because, in the current study, the release of cyto-c into the perimitochondrial domain was always associated with abnormal-pathological mitochondria, indicative of some form of subcellular perturbation. That this mitochondrial release of cyto-c is of consequence for the traumatically injured axon is supported by the finding of concomitant caspase activation whose spatial and temporal course directly paralleled cyto-c release. Although this constitutes the first description of cyto-c release and concomitant caspase activation in the axon, these interactive processes and their damaging sequelae are well ap- 

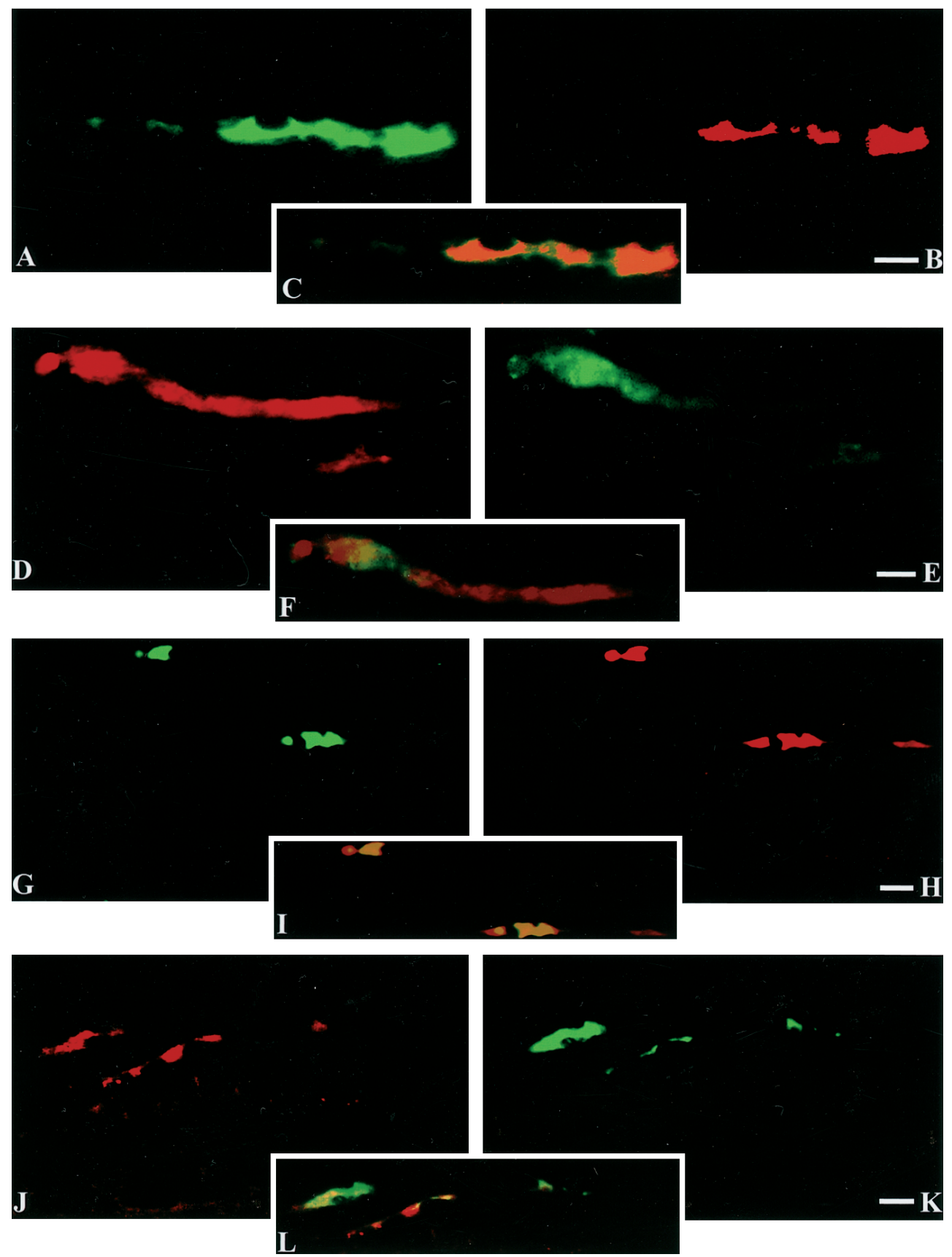

Figure 6. $A-C$, Images of $\mathrm{Ab38}(A)$ and cyto-c $(B)$ immunofluorescent damaged axonal foci and a digital overlay of the same fields $(C)$ in rat medial longitudinal fasciculus at $60 \mathrm{~min}$ after injury. Note the segmental swelling and focal vacuolization and that cyto-c and CMSP immunoreactivity are clearly colocalized in this damaged axonal segment. $D-F$, Images demonstrating cyto-c $(D)$ and caspaseassociated SBDP-120 (E) immunofluorescence damaged axonal foci and a digital overlay of the same fields $(F)$ in rat MLF at $3 \mathrm{hr}$ after injury. The morphology of the damaged immunoreactive axonal segment is consistent with imminent axonal disconnection. Again, note the obvious colocalization of the immunohistochemical markers. $G-I$, One hour after injury, immunoreactivity representing the activated form of caspase-3 enzyme $(G)$ and caspase-linked spectrin proteolysis-IR (SBDP-120) $(H)$ are directly colocalized within the same vacuolated, swollen axonal profiles $(I)$, providing direct evidence of the contribution of the caspase death cascade to the pathogenesis of traumatic axonal injury. $J-L$, Images demonstrating calpain-mediated spectrin proteolysis immunoreactivity $(J)$ and caspase-linked SBDP-120-IR $(K) 3$ hr after injury. The digital overlay of the same fields $(L)$ proves that both cysteine proteases are activated within the same, severely damaged axonal segments. Scale bar, $20 \mu \mathrm{m}$. preciated in the neuronal cell body in both in vivo and in vitro studies. Specifically, in the neuronal somata, the release of cyto-c has been shown to be pivotal in the induction of proapoptotic processes: cyto-c [formerly referred to as apoptosis proteaseactivating factor-2 (Apaf-2)] in conjunction with Apaf-1 [containing a caspase recruitment domain]) activates caspase-9, initiating the caspase death cascade, which leads to the induction of caspase-3, the primary effector enzyme in neuronal apoptosis (Thornberry and Lazebnik, 1998; Wang, 2000). In such processes, other factors can also be coreleased with cyto-c, such as caspase-2, -3, and -9 (Krajewski et al., 1999; Susin et al., 1999a) and apoptosis-inducing factor, a $57 \mathrm{kDa}$ flavoprotein capable of directly activating caspase-3 (Susin et al., 1999b).

In the current study, little consideration was given to any parallel changes occurring within the neuronal somata related to the traumatically injured axons. As is noted in the text, cyto-c or caspase immunoreactivity was not typically noted in these neurons. However, given our current understanding of the temporal course of cyto-c release and caspase perturbation in the progression of neuronal injury, this result is not surprising. It is well recognized in both the ischemic and traumatic brain injury literature that the neuronal somatic activation of cyto-c and/or caspase- 3 is a relatively delayed event, occurring at later time points than those assessed in the current communication $(\mathrm{Fu}-$ jimura et al., 1998; Pike et al., 1998a).

That the observed caspase activity was a key factor in the subsequent demise of the axon is supported by several lines of evidences. First, in the current communication, this caspase induction was recognized by two independent markers, one which directly assessed the ability of caspase to cleave intraaxonal 
spectrin (antibody targeting the SBDP-120) and the other directly targeting the active form of the capsase- 3 enzyme, providing irrefutable evidence for the activation of the caspase cascade. Second, because this caspase activity was also temporally and spatially correlated with continuing devastating intraaxonal cytoskeletal and organelle alteration, there is little doubt that these factors were participating in the agonal events and ultimate demise of the axon. These findings are entirely consistent with the observations of others who have demonstrated that, besides destroying other structural proteins, caspase-3 can irreversibly cleave the spectrin molecule (Wang et al., 1998a), with ultimate destruction of the cortical cytoskeleton, an event considered by many as the major factor in the neuronal degeneration seen in various CNS disorders (Siman et al., 1989; Saatman et al., 1996; Bartus, 1997; Bartus et al., 1998; Wang et al., 1998a). Furthermore, caspase- 3 has also been reported to cleave calpastatin, an inhibitor of calpain that might further enhance-moderate the ongoing calpain-mediated axonal demise (Wang et al., 1998b; Wood and Newcomb, 1999).

In addition to providing insight into the evolving pathobiology of traumatically induced axonal change and those factors specifically involved in its initiation and demise, we believe that the current communication is of interest because it provides unique insight in the multiplex role of cysteine proteases in the axonal pathology. Specifically, the results of the present communication, together with previously published reports from our laboratory (Büki et al., 1999a), clearly demonstrate a spatially and temporally specific cascade wherein the early activation of the calciumactivated cysteine protease calpain triggers intracellular changes involving the mitochondria with cyto-c to activate the other cysteine protease, caspase, which participates in the demise of the axon via the processes described above. From a neuropathological perspective, one could then argue the CMSP is a relatively early event (15-30 min after injury), whereas cyto-c release and caspase induction are more delayed (60-360 min), constituting terminal events. Although we believe that our data clearly indicates this, some axons in the current study did not behave in this manner, as in isolated instances, scattered axons did show early caspase activation. Although this finding would seem to compromise our premise, these isolated findings are entirely consistent with the complex nature of TBI-induced axonal injury wherein some axons sustain suprathreshold shear and tensile injury, with induction of catastrophic and ultrarapid pathological change (Maxwell et al., 1993; Büki et al., 1999a).

Although we believe that the current communication is of considerable significance for understanding the pathogenesis of traumatic brain injury, we also believe that these findings are of general interest for investigators involved in various aspects of CNS injury, such as trauma, stroke, and seizure. This statement is based on the fact that the current communication provides for the first time direct ultrastructural evidence that cyto-c release reported by others in various experimental settings $(\mathrm{Fu}-$ jimura et al., 1998; Fujimura et al., 1999; Morita-Fujimura et al., 1999 ) is directly related to the mitochondrial perturbation. We believe that our studies in the axon provide a better model than found in the neuronal somata in that we were able to examine the precise interrelationship of cyto-c release and mitochondrial damage in a discrete, isolated axonal segment of only a few micrometers in diameter. This is in distinct contrast to the situation involving the neuronal soma in which one must deal with thousands of mitochondria in a somatic domain that can exceed several thousand cubic micrometers, making any direct correla- tion between the release of cyto-c and mitochondrial damage more tenable.

In light of the above findings, the obvious question remains as to what implications they have for better understanding the pathobiology and more importantly the treatment of traumatically induced axonal injury. Although we have now defined distinct progression of calcium-induced, calpain-mediated intraaxonal proteolysis, cyto-c release, and caspase activation, it remains to be seen which of these constitutes the best target for potential therapeutic intervention in either animals or humans. No direct data argues for one therapy versus the other, yet the distinct spatial and temporal progression of events described in the current study suggests that potentially beneficial therapy would most likely act on a target, before the mitochondrial release of cyto-c. This is based on the fact that mitochondrial cyto-c release indicates severe disturbances in electron transport and the generation of free radicals (Cai and Jones, 1998; Reed, 1998; Saikumar et al., 1998). Furthermore, caspase activation as a direct consequence of mitochondrial alteration leads to irreversible structural damage of the axon. Thus, the overall consequences of cyto-c release appear so devastating as to not be amenable to therapeutic intervention. In contrast, the earlier phases of TAI involving the calciumactivated neutral proteases and perhaps the induction of MPT would seen more rational targets for therapeutic intervention. Although we have no direct experience with calpain inhibitors, we would note that several experimental studies have reported their potential efficacy in the case of TBI (Saatman et al., 1996b; Posmantur et al., 1997; Bartus et al., 1999). In these cases, however, the therapeutic targets were not axons, but rather neuronal soma taken from contusional sites. That MPT would also constitute an appropriate target for therapeutic intervention is also supported by the finding that the use of inhibitors of the mitochondrial permeability transition, which preserve mitochondria, also translate into significant axonal protection. This indicates that the mitochondrial protection itself may also provide axonal protection (Büki et al., 1999b; Okonkwo and Povlishock, 1999; Okonkwo et al., 1999).

In summary, we believe that the current communication provides a relatively complete pathobiological description of those intraaxonal events and their biological triggers that translate into irreversible axonal damage in TBI. These studies significantly extend our understanding of the pathobiology of TBI and provide unique insight into the rationale for the use of various therapeutic interventions targeting TAI.

\section{REFERENCES}

Adams H, Mitchell DE, Graham DI, Doyle D (1977) Diffuse brain damage of immediate impact type. Its relationship to "primary brainstem damage" in head injury. Brain 100:489-502.

Adams JH (1982) Diffuse axonal injury in non-missile head injury. Injury $13: 444-445$.

Adams JH, Graham DI, Scott G, Parker LS, Doyle D (1980) Brain damage in fatal non-missile head injury. J Clin Pathol 33:1132-1145.

Bartus RT (1997) The calpain hypothesis of neurodegeneration: evidence for a common cytotoxic pathway. The Neuroscientist 3:314-327.

Bartus RT, Dean RL, Mennerick S, Eveleth D, Lynch G (1998) Temporal ordering of pathogenic events following transient global ischemia. Brain Res 790:1-13.

Bartus RT, Chen EY, Lynch G, Kordower JH (1999) Cortical ablation induces a spreading calcium-dependent, secondary pathogenesis which can be reduced by inhibiting calpain. Exp Neurol 155:315-326.

Büki A, Siman R, Trojanowski JQ, Povlishock JT (1999a) The role of calpain-mediated spectrin proteolysis in traumatically induced axonal injury. J Neuropathol Exp Neurol 58:365-375.

Büki A, Okonkwo DO, Povlishock JT (1999b) Postinjury cyclosporin a 
administration limits axonal damage and disconnection in traumatic brain injury. J Neurotrauma 16:511-521.

Büki A, Koizumi H, Povlishock JT (1999c) Moderate posttraumatic hypothermia decreases early calpain-mediated proteolysis and concomitant cytoskeletal compromise in traumatic axonal injury. Exp Neurol 159:319-328.

Büki A, Walker SA, Stone JR, Povlishock JT (2000) Novel application of the tyramide signal amplification (TSA): ultrastructural visualization of double labeled immunofluorescent axonal profiles. J Histochem Cytochem 48:153-161.

Cai J, Jones DP (1998) Superoxide in apoptosis. Mitochondrial generation triggered by cytochrome c loss. J Biol Chem 273:11401-11404.

Cai J, Yang J, Jones DP (1998) Mitochondrial control of apoptosis: the role of cytochrome c. Biochim Biophys Acta 1366:139-149.

Clark RS, Chen J, Watkins SC, Kochanek PM, Chen M, Stetler RA, Loeffert JE, Graham SH (1997) Apoptosis-suppressor gene bcl-2 expression after traumatic brain injury in rats. J Neurosci 17:9172-9182.

Conti AC, Raghupathi R, Trojanowski JQ, McIntosh TK (1998) Experimental brain injury induces regionally distinct apoptosis during the acute and delayed post-traumatic period. J Neurosci 18:5663-5672.

Eskes R, Antonsson B, Osen-Sand A, Montessuit S, Richter C, Sadoul R, Mazzei G, Nichols A, Martinou JC (1998) Bax-induced cytochrome C release from mitochondria is independent of the permeability transition pore but highly dependent on $\mathrm{Mg}^{2+}$ ions. J Cell Biol 143:217-224.

Foda MA, Marmarou A (1994) A new model of diffuse brain injury in rats. II. Morphological characterization. J Neurosurg 80:301-313.

Fox GB, Fan L, LeVasseur RA, Faden AI (1998) Sustained sensory/ motor and cognitive deficits with neuronal apoptosis following controlled cortical impact brain injury in the mouse. J Neurotrauma 15:599-614.

Fujimura M, Morita-Fujimura Y, Murakami K, Kawase M, Chan PH (1998) Cytosolic redistribution of cytochrome c after transient focal cerebral ischemia in rats. J Cereb Blood Flow Metab 18:1239-1247.

Fujimura M, Morita-Fujimura Y, Kawase M, Copin JC, Calagui B, Epstein CJ, Chan PH (1999) Manganese superoxide dismutase mediates the early release of mitochondrial cytochrome $\mathrm{C}$ and subsequent DNA fragmentation after permanent focal cerebral ischemia in mice. J Neurosci 19:3414-3422.

Gennarelli TA, Thibault LE, Adams JH, Graham DI, Thompson CJ, Marcincin RP (1982) Diffuse axonal injury and traumatic coma in the primate. Ann Neurol 12:564-574.

Goodman SR, Zimmer WE, Clark MB, Zagon IS, Barker JE, Bloom ML (1995) Brain spectrin: of mice and men. Brain Res Bull 36:593-606.

Gorman AM, Bonfoco E, Zhivotovsky B, Orrenius S, Ceccatelli S (1999) Cytochrome c release and caspase-3 activation during colchicineinduced apoptosis of cerebellar granule cells. Eur J Neurosci 11:1067-1072.

Hirsch T, Susin SA, Marzo I, Marchetti P, Zamzami N, Kroemer G (1998) Mitochondrial permeability transition in apoptosis and necrosis. Cell Biol Toxicol 14:141-145.

Hunyady B, Krempels K, Harta G, Mezey E (1996) Immunohistochemical signal amplification by catalyzed reporter deposition and its application in double immunostaining. J Histochem Cytochem 44:1353-1362.

Jurgensmeier JM, Xie Z, Deveraux Q, Ellerby L, Bredesen D, Reed JC (1998) Bax directly induces release of cytochrome c from isolated mitochondria. Proc Natl Acad Sci USA 95:4997-5002.

Krajewski S, Krajewska M, Ellerby LM, Welsh K, Xie Z, Deveraux QL, Salvesen GS, Bredesen DE, Rosenthal RE, Fiskum G, Reed JC (1999) Release of caspase-9 from mitochondria during neuronal apoptosis and cerebral ischemia. Proc Natl Acad Sci USA 96:5752-5757.

Lemasters JJ, Nieminen AL, Qian T, Trost LC, Elmore SP, Nishimura Y, Crowe RA, Cascio WE, Bradham CA, Brenner DA, Herman B (1998) The mitochondrial permeability transition in cell death: a common mechanism in necrosis, apoptosis and autophagy. Biochim Biophys Acta 1366:177-196.

Marmarou A, Foda MA, van den Brink W, Campbell J, Kita H, Demetriadou K (1994) A new model of diffuse brain injury in rats. I. Pathophysiology and biomechanics. J Neurosurg 80:291-300.

Martinou I, Desagher S, Eskes R, Antonsson B, Andre F, Fakan S, Martinou JC (1999) The release of cytochrome c from mitochondria during apoptosis of NGF-deprived sympathetic neurons is a reversible event. J Cell Biol 144:883-889.

Maxwell WL, Watt C, Graham DI, Gennarelli TA (1993) Ultrastructural evidence of axonal shearing as a result of lateral acceleration of the head in non-human primates. Acta Neuropathol (Berl) 86:136-144.

Maxwell WL, Povlishock JT, Graham DL (1997) A mechanistic analysis of nondisruptive axonal injury: a review. J Neurotrauma [Erratum (1997) 14:755] 14:419-440.

McIntosh TK, Saatman KE, Raghupathi R, Graham DI, Smith DH, Lee VM, Trojanowski JQ (1998) The Dorothy Russell memorial lecture. The molecular and cellular sequelae of experimental traumatic brain injury: pathogenetic mechanisms. Neuropathol Appl Neurobiol 24:251-267.

Montal M (1998) Mitochondria, glutamate neurotoxicity and the death cascade. Biochim Biophys Acta 1366:113-126.

Morita-Fujimura Y, Fujimura M, Kawase M, Chen SF, Chan PH (1999) Release of mitochondrial cytochrome $\mathrm{c}$ and DNA fragmentation after cold injury-induced brain trauma in mice: possible role in neuronal apoptosis. Neurosci Lett 267:201-205.

Nath R, Probert Jr A, McGinnis KM, Wang KK (1998) Evidence for activation of caspase-3-like protease in excitot. J Neurochem 71:186-195.

Okonkwo DO, Povlishock JT (1999) An intrathecal bolus of cyclosporin A before injury preserves mitochondrial integrity and attenuates axonal disruption in traumatic brain injury. J Cereb Blood Flow Metab 19:443-451.

Okonkwo DO, Büki A, Siman R, Povlishock JT (1999) Cyclosporin A limits calcium-induced axonal damage following traumatic brain injury. NeuroReport 10:353-358.

Petrusz P, Sar M, Ordronneau P, DiMeo P (1976) Specificity in immunocytochemical staining. J Histochem Cytochem 24:1110-1112.

Petrusz P, Ordronneau P, Finley JC (1980) Criteria of reliability for light microscopic immunocytochemical staining. Histochem J 12:333-348.

Pettus EH, Christman CW, Giebel ML, Povlishock JT (1994) Traumatically induced altered membrane permeability: its relationship to traumatically induced reactive axonal change. J Neurotrauma 11:507-522.

Pike BR, Zhao X, Newcomb JK, Wang KK, Posmantur RM, Hayes RL (1998a) Temporal relationships between de novo protein synthesis, calpain and caspase 3-like protease activation, and DNA fragmentation during apoptosis in septo-hippocampal cultures. J Neurosci Res 52:505-520.

Pike BR, Zhao X, Newcomb JK, Posmantur RM, Wang KK, Hayes RL (1998b) Regional calpain and caspase-3 proteolysis of alpha-spectrin after traumatic brain injury. NeuroReport 9:2437-2442.

Posmantur R, Kampfl A, Siman R, Liu J, Zhao X, Clifton GL, Hayes RL (1997) A calpain inhibitor attenuates cortical cytoskeletal protein loss after experimental traumatic brain injury in the rat. Neuroscience 77:875-888.

Povlishock JT (1992) Traumatically induced axonal injury: pathogenesis and pathobiological implications. Brain Pathol 2:1-12.

Povlishock JT, Pettus EH (1996) Traumatically induced axonal damage: evidence for enduring changes in axolemmal permeability with associated cytoskeletal change. Acta Neurochir Suppl (Wien) 66:81-86.

Povlishock JT, Becker DP, Cheng CL, Vaughan GW (1983) Axonal change in minor head injury. J Neuropathol Exp Neurol 42:225-242.

Povlishock JT, Marmarou A, McIntosh T, Trojanowski JQ, Moroi J (1997) Impact acceleration injury in the rat: evidence for focal axolemmal change and related neurofilament sidearm alteration. J Neuropathol Exp Neurol 56:347-359.

Reed JC (1998) Bcl-2 family proteins. Oncogene 17:3225-3236.

Rink A, Fung KM, Trojanowski JQ, Lee VM, Neugebauer E, McIntosh TK (1995) Evidence of apoptotic cell death after experimental traumatic brain injury in the rat. Am J Pathol 147:1575-1583.

Roberts-Lewis JM, Savage MJ, Marcy VR, Pinsker LR, Siman R (1994) Immunolocalization of calpain I-mediated spectrin degradation to vulnerable neurons in the ischemic gerbil brain. J Neurosci 14:3934-3944.

Saatman KE, Bozyczko-Coyne D, Marcy V, Siman R, McIntosh TK (1996a) Prolonged calpain-mediated spectrin breakdown occurs regionally following experimental brain injury in the rat. J Neuropathol Exp Neurol 55:850-860.

Saatman KE, Murai H, Bartus RT, Smith DH, Hayward NJ, Perri BR, McIntosh TK (1996b) Calpain inhibitor AK295 attenuates motor and cognitive deficits following experimental brain injury in the rat. Proc Natl Acad Sci USA 93:3428-3433.

Saikumar P, Dong Z, Weinberg JM, Venkatachalam MA (1998) Mechanisms of cell death in hypoxia/reoxygenation injury. Oncogene 17:3341-3349. 
Schendel SL, Montal M, Reed JC (1998) Bcl-2 family proteins as ionchannels. Cell Death Differ 5:372-380.

Siesjo BK, Hu B, Kristian T (1999) Is the cell death pathway triggered by the mitochondrion or the endoplasmic reticulum? J Cereb Blood Flow Metab 19:19-26.

Siman R, Noszek JC, Kegerise C (1989) Calpain I activation is specifically related to excitatory amino acid induction of hippocampal damage. J Neurosci 9:1579-1590.

Stone JR, Walker SA, Povlishock JT (1999) The visualization of a new class of traumatically injured axons through the use of a modified method of microwave antigen retrieval. Acta Neuropathol (Berl) 97:335-345.

Strich SJ (1956) Diffuse degeneration of the cerebral white matter in severe dementia following head injury. J Neurol Neurosurg Psychiatry 19:163-185.

Strich SJ (1961) Shearing of nerve fibers as a cause of brain damage due to head injury: a pathological study of twenty cases. Lancet 2:443-448.

Susin SA, Zamzami N, Kroemer G (1998) Mitochondria as regulators of apoptosis: doubt no more. Biochim Biophys Acta 1366:151-165.

Susin SA, Lorenzo HK, Zamzami N, Marzo I, Brenner C, Larochette N, Prevost MC, Alzari PM, Kroemer G (1999a) Mitochondrial release of caspase-2 and -9 during the apoptotic process. J Exp Med 189:381-394.

Susin SA, Lorenzo HK, Zamzami N, Marzo I, Snow BE, Brothers GM, Mangion J, Jacotot E, Costantini P, Loeffler M, Larochette N, Goodlett DR, Aebersold R, Siderovski DP, Penninger JM, Kroemer G (1999b) Molecular characterization of mitochondrial apoptosis-inducing factor. Nature 397:441-446.

Thornberry NA, Lazebnik Y (1998) Caspases: enemies within. Science 281:1312-1316.

Trost LC, Lemasters JJ (1996) The mitochondrial permeability transition: a new pathophysiological mechanism for Reye's syndrome and toxic liver injury. J Pharmacol Exp Ther 278:1000-1005.

Uehara T, Kikuchi Y, Nomura Y (1999) Caspase activation accompa- nying cytochrome c release from mitochondria is possibly involved in nitric oxide-induced neuronal apoptosis in SH-SY5Y cells. J Neurochem 72:196-205.

van Gijlswijk RP, Zijlmans HJ, Wiegant J, Bobrow MN, Erickson TJ, Adler KE, Tanke HJ, Raap AK (1997) Fluorochrome-labeled tyramides: use in immunocytochemistry and fluorescence in situ hybridization. J Histochem Cytochem 45:375-382.

Wang KK (2000) Calpain and caspase: can you tell the difference? Trends Neurosci 23:20-26.

Wang KK, Posmantur R, Nath R, McGinnis K, Whitton M, Talanian RV, Glantz SB, Morrow JS (1998a) Simultaneous degradation of alphaIIand betaII-spectrin by caspase 3 (CPP32) in apoptotic cells. J Biol Chem 273:22490-22497.

Wang KK, Posmantur R, Nadimpalli R, Nath R, Mohan P, Nixon RA, Talanian RV, Keegan M, Herzog L, Allen H (1998b) Caspasemediated fragmentation of calpain inhibitor protein calpastatin during apoptosis. Arch Biochem Biophys 356:187-196.

Wood DE, Newcomb EW (1999) Caspase-dependent activation of calpain during drug-induced apoptosis. J Biol Chem 274:8309-8315.

Yakovlev AG, Knoblach SM, Fan L, Fox GB, Goodnight R, Faden AI (1997) Activation of CPP32-like caspases contributes to neuronal apoptosis and neurological dysfunction after traumatic brain injury. J Neurosci 17:7415-7424.

Zagon IS, Higbee R, Riederer BM, Goodman SR (1986) Spectrin subtypes in mammalian brain: an immunoelectron microscopic study. J Neurosci 6:2977-2986.

Zhao X, Pike BR, Newcomb JK, Wang KK, Posmantur RM, Hayes RL (1999) Maitotoxin induces calpain but not caspase-3 activation and necrotic cell death in primary septo-hippocampal cultures. Neurochem Res 24:371-382.

Zoratti M, Szabo I (1995) The mitochondrial permeability transition. Biochim Biophys Acta 1241:139-176. 